\title{
S-Layer Glycoprotein From Lactobacillus kefiri Exerts Its Immunostimulatory Activity Through Glycan Recognition by Mincle
}

\author{
Mariano Malamud ${ }^{1,2}$, Paula Carasi ${ }^{1,3}$, Matías H. Assandri ${ }^{1}$, Teresa Freire ${ }^{4}$, \\ Bernd Lepenies $^{2 *}$ and María de los Ángeles Serradell ${ }^{1,5 *}$
}

${ }^{1}$ Cátedra de Microbiología, Departamento de Ciencias Biológicas, Facultad de Ciencias Exactas, Universidad Nacional de La Plata, La Plata, Argentina, ${ }^{2}$ Immunology Unit \& Research Center for Emerging Infections and Zoonoses (RIZ), University of Veterinary Medicine Hannover, Hanover, Germany, ${ }^{3}$ CCT La Plata, CONICET, Instituto de Estudios Inmunológicos y Fisiopatológicos (IIFP), La Plata, Argentina, ${ }^{4}$ Laboratorio de Inmunomodulación y Desarrollo de Vacunas, Departamento de Inmunobiología, Facultad de Medicina, Universidad de la República, Montevideo, Uruguay, ${ }^{5}$ Instituto de Ciencias de la Salud, Universidad Arturo Jauretche, Florencio Varela, Argentina

OPEN ACCESS

Edited by:

Sandip D. Kamath,

James Cook University, Australia

Reviewed by:

Ashu Sharma,

University at Buffalo, United States

Salvador Iborra

Complutense University of Madrid,

Spain

Richard Weiss,

University of Salzburg, Austria

*Correspondence:

Bernd Lepenies

bernd.lepenies@tiho-hannover.de

María de los Ángeles Serradell

maserr@biol.un/p.edu.ar

Specialty section:

This article was submitted to Vaccines and Molecular Therapeutics, a section of the journal

Frontiers in Immunology

Received: 27 March 2019 Accepted: 05 June 2019

Published: 26 June 2019

Citation:

Malamud M, Carasi P, Assandri MH, Freire T, Lepenies B and Serradell MÁ

(2019) S-Layer Glycoprotein From

Lactobacillus kefiri Exerts Its Immunostimulatory Activity Through

Glycan Recognition by Mincle.

Front. Immunol. 10:1422

doi: 10.3389/fimmu.2019.01422
The development of new subunit vaccines has promoted the rational design of adjuvants able to induce a strong T-cell activation by targeting specific immune receptors. The S-layer is a (glyco)-proteinaceous envelope constituted by subunits that self-assemble to form a two-dimensional lattice that covers the surface of different species of Bacteria and Archaea. Due to their ability to self-assemble in solution, they are attractive tools to be used as antigen/hapten carriers or adjuvants. Recently, we have demonstrated that S-layer glycoprotein from Lactobacillus kefiri CIDCA 8348 (SLP-8348) enhanced the LPS-induced response on macrophages in a $\mathrm{Ca}^{2+}$-dependent manner, but the receptors involved in these immunomodulatory properties remain unknown. Therefore, we aim to determine the C-type lectin receptors (CLRs) recognizing this bacterial surface glycoprotein as well as to investigate the role of glycans in both the immunogenicity and adjuvant capacity of SLP-8348. Here, using a mild periodate oxidation protocol, we showed that loss of SLP-8348 glycan integrity impairs the cell-mediated immune response against the protein. Moreover, our data indicate that the adjuvant capacity of SLP-8348 is also dependent of the biological activity of the SLP-8348 glycans. In order to evaluate the CLRs involved in the interaction with SLP-8348 an ELISA-based method using CLR-hFc fusion proteins showed that SLP-8348 interacts with different CLRs such as Mincle, SingR3, and hDC-SIGN. Using BMDCs derived from CLR-deficient mice, we show that SLP-8348 uptake is dependent of Mincle. Furthermore, we demonstrate that the SLP-8348-induced activation of BMDCs as well as its adjuvant capacity relies on the presence of Mincle and its signaling adaptor CARD9 on BMDCs, since SLP-8348-activated BMDCs from Mincle $^{-/-}$or $\mathrm{CARD9}^{-/-}$mice were not capable to enhance OVA-specific response in $\mathrm{CD}^{+}{ }^{+} \mathrm{T}$ cells purified from OT-II mice. These findings significantly contribute to the understanding of the role of glycans in the immunomodulation elicited by bacterial SLPs and generate a great opportunity in the search for new adjuvants derived from non-pathogenic microorganisms.

Keywords: S-layer protein, adjuvants, C-type lectin receptors, DCs activation, lactobacillus 


\section{INTRODUCTION}

Studies of the biotechnological applications of S-layer proteins, considered as one of the most abundant biopolymers on our planet (1), have been increasing in recent years. The S-layer is a surface macromolecular array, found on both Grampositive and Gram-negative bacteria and highly prevalent in archaea (2). It is generally formed by identical protein or glycoprotein subunits that are held together by non-covalent interactions and self-assemble to form a two-dimensional lattice $(3,4)$. Since the S-layer constitutes the outermost surface structure in those microorganisms, it is in direct contact with the bacterial environment and could mediate interactions with host cells. This feature, together with the ability to self-assemble and its unique physicochemical properties, make S-layer proteins (SLPs) an attractive tool to use as antigen/hapten carrier, as adjuvant, or as part of vaccination vesicles (1). Therefore, understanding how SLPs interact with immune cells is a critical step toward the application of these proteins in the development of new adjuvanted vaccines.

It is known that upon recognition of microbial structures through different pattern recognition receptors (PRRs), antigen presenting cells (APCs) such as dendritic cells (DCs) and macrophages, undergo signal transduction that lead to cell maturation with the up-regulation of co-stimulatory molecules and production of different chemokines and cytokines. There are different classes of PRR families, including transmembrane proteins such as Toll-like receptors (TLRs) and C-type lectin receptors (CLRs) (5). TLRs are a family of membrane-bound proteins that mainly recognize microbial membrane components such as lipids, lipoproteins, and proteins (6). In contrast, CLRs are specialized in the recognition of carbohydrates, through one or more carbohydrate recognition domains (CRDs). Depending on the receptors engaged, APCs display different maturation states and produce different inflammatory mediators that impact the following cellular and humoral responses $(7,8)$.

Lactobacillus kefiri is a lactic acid bacterium derived from kefir, carrying an S-layer glycoprotein in its envelope (9-11). It has been shown that $L$. kefiri has immunomodulatory properties, and both in vitro and in vivo experiments support its potential as a probiotic microorganism $(12,13)$. Moreover, the SLPs from $L$. kefiri antagonize the effect of Clostridium difficile toxins on Vero cells (14), mediate the inhibition of Salmonella enterica invasion to Caco-2 cells (15) and they also enhance the adhesion of L. kefiri to gastrointestinal mucus (16).

Glycosylation is the most frequent post-translational modification found on SLPs (17). Some studies have been conducted to address the role of the glycans present in the SLPs from different Lactobacillus species in their immunomodulatory properties. In LPS-treated immature dendritic cells, the SLP from L. acidophilus NCFM was able to induce an anti-inflammatory profile, mediated by the CLR DC-specific ICAM-3-grabbing non-integrin (DC-SIGN) (18). Moreover, this SLP-DC-SIGN engagement was demonstrated for S-layer like proteins from $L$. plantarum (19) and was also suggested for the SLP from L. kefiri JCM 5818 (20).
Recently, we have demonstrated that the S-layer glycoprotein from Lactobacillus kefiri CIDCA 8348 (SLP-8348) was internalized by macrophages in a process that was mediated by carbohydrate-receptor interactions. In addition, SLP-8348 enhanced the LPS-induced response on macrophages in a $\mathrm{Ca}^{+2}$ dependent manner (21). However, the molecular mechanisms as well as the receptors involved in the immunomodulatory properties elicited by SLP-8348 on APCs are not well-understood. Thus, the aim of this work was to investigate the involvement of CLRs in the immune cell response to SLP-8348 using in vitro and in vivo approaches.

\section{MATERIALS AND METHODS}

\section{Bacterial Strains and Growth Conditions}

L. kefiri CIDCA 8348 isolated from kefir grains was used (22). The strain was cultured in de Man-Rogosa-Sharpe (MRS) broth (Difco, Beauvais, France) at $37^{\circ} \mathrm{C}$ for $48 \mathrm{~h}$ in aerobic conditions. Frozen stock cultures were stored at $-80^{\circ} \mathrm{C}$ in skim milk until use.

\section{S-Layer Protein Extraction}

S-layer protein extraction from bacterial cells at stationary phase was performed using $5 \mathrm{M} \mathrm{LiCl}$ as previously described (14). SLPs extracts were tested by sodium dodecylsulphate-polyacrylamide gel electrophoresis (SDS-PAGE) in 12\% separating and $4 \%$ stacking gels using the discontinuous buffer system according to Laemmli (23). Gels were migrated on a BioRad Mini-Protean II (BioRad Laboratories, Richmond, CA, USA) and revealed using Colloidal Blue Staining. Carbohydrate glycol groups present in SLP-8348 were oxidized with sodium periodate $(10 \mathrm{mM})$ as previously described by Rodriguez et al (24). The oxidation was performed at room temperature for $45 \mathrm{~min}$ in the dark, followed by the reduction with sodium borohydride $(50 \mathrm{mM})$ of the reactive aldehyde groups (24). The resulting oxidized SLP is referred as SLPOx-8348. The control, SLPB-8348, consisted of SLP-8348 subjected to the whole treatment excepting for the incubation with sodium periodate. SLPs were dialyzed against PBS and then filtrated through a membrane of $0.45 \mu \mathrm{m}$ pore diameter. Protein concentration was determined according to Bradford (25).

\section{Cell Cultures}

The monocyte/macrophage murine cell line RAW 264.7 was cultured in Dulbecco's Modified Eagle Medium (DMEM) supplemented with: $10 \%(\mathrm{v} / \mathrm{v})$ heat-inactivated $\left(30 \mathrm{~min} / 60^{\circ} \mathrm{C}\right)$ fetal bovine serum (FBS), $1 \%(\mathrm{v} / \mathrm{v})$ non-essential amino acids and $1 \%(\mathrm{v} / \mathrm{v})$ penicillin-streptomycin solution $(100 \mathrm{U} / \mathrm{mL}$ penicillin $\mathrm{G}, 100 \mathrm{~g} / \mathrm{mL}$ streptomycin). All cell culture reagents were from GIBCO BRL Life Technologies (Rockville, MD, USA).

BMDCs were generated from C57BL/ 6 wild type, Mincle ${ }^{-/-}$, CARD9 ${ }^{-/-}$, or SignR3 ${ }^{-/-}$bone marrow precursors $\left(2.5 \times 10^{5}\right.$ cells $/ \mathrm{ml}$ ) that were plated in complete culture medium (IMDM supplemented with $2 \mathrm{mM}$ glutamine, $10 \%$ (v/v) FBS, $100 \mathrm{U} / \mathrm{ml}$ penicillin and $100 \mu \mathrm{g} / \mathrm{ml}$ streptomycin) supplemented with a GM-CSF containing supernatant from P3-X63 cells. Medium was exchanged every $48 \mathrm{~h}$ and BMDCs were used after 8-10 days 
of differentiation to ascertain that $\geq 80 \%$ of the cell population expressed the marker CD11c.

\section{Binding and Internalization of SLP-8348 by BMDCs}

Binding and internalization of SLP-8348 were analyzed by flow cytometry. Labeling of SLP-8348 with Atto $647 \mathrm{~N}$ was performed according to the manufacturer's instructions (SIGMA, USA). BMDCs from C57BL/6 mice, Mincle-deficient and SignR3deficient mice $\left(2.5 \times 10^{5} / \mathrm{mL}\right)$ were incubated with Atto 647 $\mathrm{N}$-labeled SLP-8348 for $1 \mathrm{~h}$ at $37^{\circ} \mathrm{C}$ (to assess uptake), or at $4^{\circ} \mathrm{C}$ in complete medium (to assess binding) $(21,24)$. Cells were then washed, incubated with mouse anti-CD11c antibody and analyzed by flow cytometry.

\section{Cells Stimulation Assays}

RAW 264.7 cells $\left(2.5 \times 10^{5}\right)$ were distributed onto 24-well microplates (JET BIOFIL ${ }^{\circledR}$, China), and the medium volume was adjusted to $0.5 \mathrm{~mL}$. The plates were incubated for $48 \mathrm{~h}$ at $37^{\circ} \mathrm{C}$ in a $5 \% \mathrm{CO}_{2} 95 \%$ air atmosphere to allow cell adherence prior to experimentation. After that, cells were treated with SLP-8348 $(10 \mu \mathrm{g} / \mathrm{mL})$, SLPOx-8348 $(10 \mu \mathrm{g} / \mathrm{mL})$ or SLPB-8348 $(10 \mu \mathrm{g} / \mathrm{mL})$ in the presence or absence of LPS $0.1 \mu \mathrm{g} / \mathrm{mL}$ (LPS from Escherichia coli O111:B4, SIGMA, USA), in DMEM for $24 \mathrm{~h}$ at $37^{\circ} \mathrm{C}$ in a $5 \% \mathrm{CO}_{2} 95 \%$ air atmosphere. Cells incubated with DMEM were used as negative control.

BMDCs from wild type or CLR-deficient mice $\left(1 \times 10^{5}\right.$ cells/well) were distributed onto 96-well microplates (Sarstedt, Germany) and stimulated with SLP-8348 $(10 \mu \mathrm{g} / \mathrm{mL})$, LPS $(0.25 \mu \mathrm{g} / \mathrm{mL})$ or the combination of both for $16 \mathrm{~h}$ at $37^{\circ} \mathrm{C}$ in a $5 \% \mathrm{CO}_{2} 95 \%$ air atmosphere. Culture supernatants were collected and analyzed by ELISA for IL- 6 and TNF- $\alpha$ secretion. Cells were incubated with anti-CD16/32 (93) to block cell surface Fc $\gamma$ RII/RIII receptors, stained with anti-CD11c (N418), CD40 (3/23), CD80 (16-10A1) and then analyzed by flow cytometry.

\section{Mice}

For in vivo experiments, 6- to 8-week-old female BALB/c mice were purchased from the Animal Care Facility of the Facultad de Ciencias Veterinarias of the Universidad Nacional de La Plata (Argentina) or DILAVE Laboratories (Uruguay). Animals were kept in the animal house (Cátedra de Microbiología, Facultad de Ciencias Exactas, UNLP, La Plata, Argentina or URBE, Facultad de Medicina, UdelaR, Montevideo, Uruguay) with water and food supplied ad libitum, and handled in accordance with institutional guidelines for animal welfare.

C57BL/6, C57BL/6-Tg (TcraTcrb)425Cbn/J mice (OT-II transgenic mice), Mincle ${ }^{-/-}$, CARD9 ${ }^{-/-}$or SignR3 ${ }^{-/-}$were kept in the animal house of the University of Veterinary Medicine (Hannover, Germany) with water and food supplied ad libitum. Mice were sacrificed for the isolation of spleen cells (OT-II transgenic mice) or the preparation of bone marrow for BMDC generation $\left(\right.$ Mincle $^{-/-}, \mathrm{CARD}^{-/-}$, SignR3 ${ }^{-/-}$, and WT mice).

\section{BMDC/T Cell Co-culture Assay}

Splenocytes were isolated from OT-II transgenic mice by flushing the spleen with complete IMDM medium. After erythrocyte lysis, cells were resuspended in MACS buffer $(0.5 \%$ (w/v) BSA, $2 \mathrm{mM}$ EDTA in PBS). T cells were obtained by magnetic-activated cell separation (MACS) using Pan T cell isolation kit II, mouse (Miltenyi Biotech, Bergisch Gladbach, Germany) according to the manufacturer's instructions. T cells were labeled with CFSE (carboxyfluorescein diacetate succinimidyl ester, Sigma-Aldrich, USA) and seeded on a 96-well round bottom culture plate $(7 \times$ $10^{4}$ cells/well). After $30 \mathrm{~min}$, BMDCs treated with OVA (Endo Grade ${ }^{\circledR}$ Ovalbumin, LIONEX GmbH, Germany) or OVA/SLP8348 were added and incubated for $72 \mathrm{~h}$. Culture supernatants were collected for IFN- $\gamma$ secretion.

\section{Immunization}

To test the SLP-8348 immunogenicity, BALB/c mice (8 weeks old, 5 mice per group) were subcutaneously injected on the base of the tail with one dose of PBS, SLP-8348 (10 $\mu \mathrm{g} /$ mouse), SLPOx-8348 (10 $\mu \mathrm{g} / \mathrm{mouse})$, SLPB-8348 (10 $\mu \mathrm{g} / \mathrm{mouse})$, OVA (10 $\mu \mathrm{g} /$ mouse) or the combinations of them, with or without IFA. After 10 days cells from inguinal lymph nodes were labeled with CFSE and stimulated in vitro with SLP-8348 $(10 \mu \mathrm{g} / \mathrm{ml})$ or OVA $(10 \mu \mathrm{g} / \mathrm{ml})$ for 5 days. Experimental protocol was approved by the Institutional Animal Care and Use Committee of the Facultad de Ciencias Exactas, Universidad Nacional de La Plata (Protocol 006-00-18).

\section{C-Type Lectin Receptor Recognition of S-Layer Protein}

The CLR-reactivity on SLP-8348 was evaluated by ELISA (26). A half-area microplate (Greiner Bio-One GmbH, Frickenhausen, Germany) was coated with $0.25 \mu \mathrm{g}$ SLP-8348, SLPOx-8348, or SLPB-8348 per well for $16 \mathrm{~h}$ at $4^{\circ} \mathrm{C}$ and blocked with $1 \%(\mathrm{w} / \mathrm{v})$ BSA (Thermo Fisher Scientific, Darmstadt, Germany) for $2 \mathrm{~h}$ at room temperature (RT). Then, $0.25 \mu \mathrm{g}$ of each CLR-hFc fusion protein in lectin-binding buffer $(50 \mathrm{mM}$ HEPES, $5 \mathrm{mM}$ $\mathrm{MgCl}_{2}$, and $5 \mathrm{mM} \mathrm{CaCl}_{2}$ ) was added and incubated for $1 \mathrm{~h}$ at RT. For inhibition assays, CLR-hFc fusion proteins were preincubated with $5 \mathrm{mM}$ of EGTA (Sigma-Aldrich, USA). After washing, HRP-conjugated anti-human IgG antibody (Dianova) was added to each well for $1 \mathrm{~h}$ at RT. Finally, plates were incubated with chromogenic substrate (o-phenylenediamine dihydrochloride substrate tablet (Thermo Fisher Scientific), $24 \mathrm{mM}$ citrate buffer, $0.04 \%$ (v/v) $\mathrm{H}_{2} \mathrm{O}_{2}, 50 \mathrm{mM}$ phosphate buffer in $\mathrm{H}_{2} \mathrm{O}$ ). The reaction was stopped with $2.0 \mathrm{M}$ sulfuric acid and the plate was read at $495 \mathrm{~nm}$ using a Multiskan Go microplate spectrophotometer (Thermo Fisher Scientific).

\section{Cytokine Quantification in Cell Culture Supernatants}

Production of IL- 6 by macrophages and IFN- $\gamma$ by cells from inguinal lymph nodes were analyzed by sandwich ELISA using commercially available capture and detection antibodies from BD-Pharmingen (San Diego, USA). Secretion of IL-6 and TNF$\alpha$ by BMDCs and IFN- $\gamma$ from purified T cells were analyzed by ELISA from PeproTech (USA). The assays were performed according to the manufacturer's instructions. After determining optical densities, cytokine levels in cell culture supernatants were calculated using the GraphPad Prism 6.0 program. 


\section{Immunocytostaining and Flow Cytometry}

After stimulation experiments, cells were washed twice with PBS containing 2\% (v/v) FBS and then labeled with specific antibodies for $30 \mathrm{~min}$ at $4^{\circ} \mathrm{C}$. Cells were washed twice with PBS containing $2 \%(\mathrm{v} / \mathrm{v}) \mathrm{FBS}$, and then fixed with $1 \%(\mathrm{v} / \mathrm{v})$ formaldehyde. Cells were analyzed using a FACSCalibur Analyzer (BD Biosciences), Accuri C6 (BD Biosciences) or Attune NxT Flow Cytometer (Thermo Fisher Scientific). Data analysis was performed using the FlowJo Software (FlowJo, Ashland, OR, USA).

\section{Statistical Analysis}

Statistical analysis was performed with the GraphPad Prism program. Values from at least three independent experiments were analyzed by using a one-way or two-way ANOVA with Tukey's post hoc test $(p<0.05$ was considered statistically significant).

\section{RESULTS}

\section{SLP-8348 Glycan Moieties Modulate the TLR-Induced Maturation of Macrophages}

Previous studies performed in our laboratory demonstrated the capacity of SLP-8348 to enhance in vitro TLR-induced maturation of murine macrophages in a $\mathrm{Ca}^{+2}$-dependent manner (21). In order to determine if the carbohydrates from SLP-8348 participate in the induction of high levels of macrophage activation, we performed a mild periodate oxidation of glycans where the glycol groups in carbohydrates are oxidized to reactive aldehyde groups, which are in turn reduced with sodium borohydride. During this process, the glycans and the integrity of glycoproteins are maintained, but instead the molecular conformation of the glycans is altered, losing possible biological activity (24). Macrophages stimulated with oxidized S-layer protein (SLPOx-8348) and LPS produced similar levels of IL-6 as well as a comparable expression of co-stimulatory molecules CD40 and CD86 as cells incubated with LPS alone (Figure 1). Macrophages incubated with the control SLPB-8348 and LPS, developed the same stimulation profile as cells in presence of LPS and SLP-8348, with higher levels of IL-6, CD40 and CD86 compared to LPS-treated macrophages (Figure 1). These results indicate that terminal glycans present on SLP-8348 determine the immunostimulatory activity on LPS-matured macrophages.

\section{Glycan Oxidation Impairs the Immunogenicity of SLP-8348}

In order to analyse SLP-8348 immunogenicity, one dose (10 $\mu \mathrm{g} /$ mouse) of SLP-8348 in combination with incomplete Freund's adjuvant (IFA) was subcutaneously injected into $\mathrm{BALB} / \mathrm{c}$ mice and antigen specific $\mathrm{T}$-cell proliferation in inguinal lymph nodes was evaluated 10 days post-injection. $\mathrm{CD}^{+} \mathrm{T}$ cells proliferation index as well as secretion of IFN- $\gamma$ were significantly higher in the group of mice treated with SLP-8348/IFA respect to PBS/IFA-treated mice, after ex vivo stimulation with SLP-8348 (Figure 2A; Supplementary Figure 1). Surprisingly, when the experiments were performed in the absence of IFA, the group of mice treated with SLP-8348 alone exhibited similar responses as SLP-8348/IFA-treated mice, indicating that SLP-8348 is a highly immunogenic glycoprotein able to induce $\mathrm{T}$ cell proliferation and cytokine production in absence of adjuvant (Figure 2A; Supplementary Figure 1).

In order to establish whether the glycan structures present on SLP-8348 participate in the induction of a cell-mediated immune response, we evaluated the T-cell proliferation in inguinal lymph nodes from mice subcutaneously injected with SLP-8348, SLPOx8348 , or SLPB-8348. CD4 ${ }^{+} \mathrm{T}$ cell proliferation index as well as secretion of IFN- $\gamma$ were significantly lower in the group of mice treated with SLPOx-8348 compared to SLP-8348-treated mice, after ex vivo stimulation with SLP-8348 (Figure 2B). The group of mice treated with SLPB-8348 displayed a similar response as SLP-8348-treated mice. These results indicate that SLP-8348 carbohydrates play an important role in the SLP-8348-induced T cell stimulation.

\section{Loss of Glycan Integrity Abrogates the Adjuvant Capacity of SLP-8348}

Having shown that immunization with SLP-8348 can induce a specific cell-mediated immune response, and the crucial role of the carbohydrates of SLP-8348 in that effect, we subsequently explored the adjuvant potential of SLP-8348 using OVA as a model antigen. In order to evaluate the in vivo adjuvant capacity of SLP-8348, we injected subcutaneously OVA or OVA/SLP-8348 on BALB/c mice and after 10 days, cells from the inguinal lymph nodes were stimulated in vitro with OVA. Proliferation index of $\mathrm{CD}^{+} \mathrm{T}$ cells as well as secretion of IFN- $\gamma$ against OVA were significantly higher in the group of mice treated with the combination of SLP-8348 and OVA compared to OVA-treated mice (Figures 3A,B). To determine if the carbohydrates from SLP-8348 participate in the enhanced response against OVA, we carried out the same experiments using the combination of SLPOx-8348 and OVA. The proliferation index of $\mathrm{CD} 4^{+} \mathrm{T}$ cells as well as secretion of IFN- $\gamma$ against OVA were significantly lower in the group of mice treated with OVA/SLPOx-8348 compared to OVA/SLP8348-treated mice (Figures 3A,B), indicating that oxidation of glycans abrogates the adjuvant capacity of SLP-8348. Levels of IFN- $\gamma$ in absence of in vitro OVA stimulation are shown as Supplementary Figure 2.

\section{SLP-8348 Interacts With Different C-Type Lectin Receptors}

In order to identify possible CLRs that recognize SLP-8348, we performed an ELISA-based method using CLR-hFc fusion proteins (26). As shown in Figure 4A, glycoconjugates from SLP-8348 strongly reacted with Mincle, as well as with other receptors such as SignR3, hDC-SIGN and mLangerin. Taking into account that all the CLRs tested in this work recognized glycan structures in a $\mathrm{Ca}^{2+}$-dependent manner, we incubated the CLR-hFc fusion protein with SLP-8348 in the presence of EGTA. We observed that around $70 \%$ of the SLP-8348/CLR-hFc binding was abrogated with EGTA 

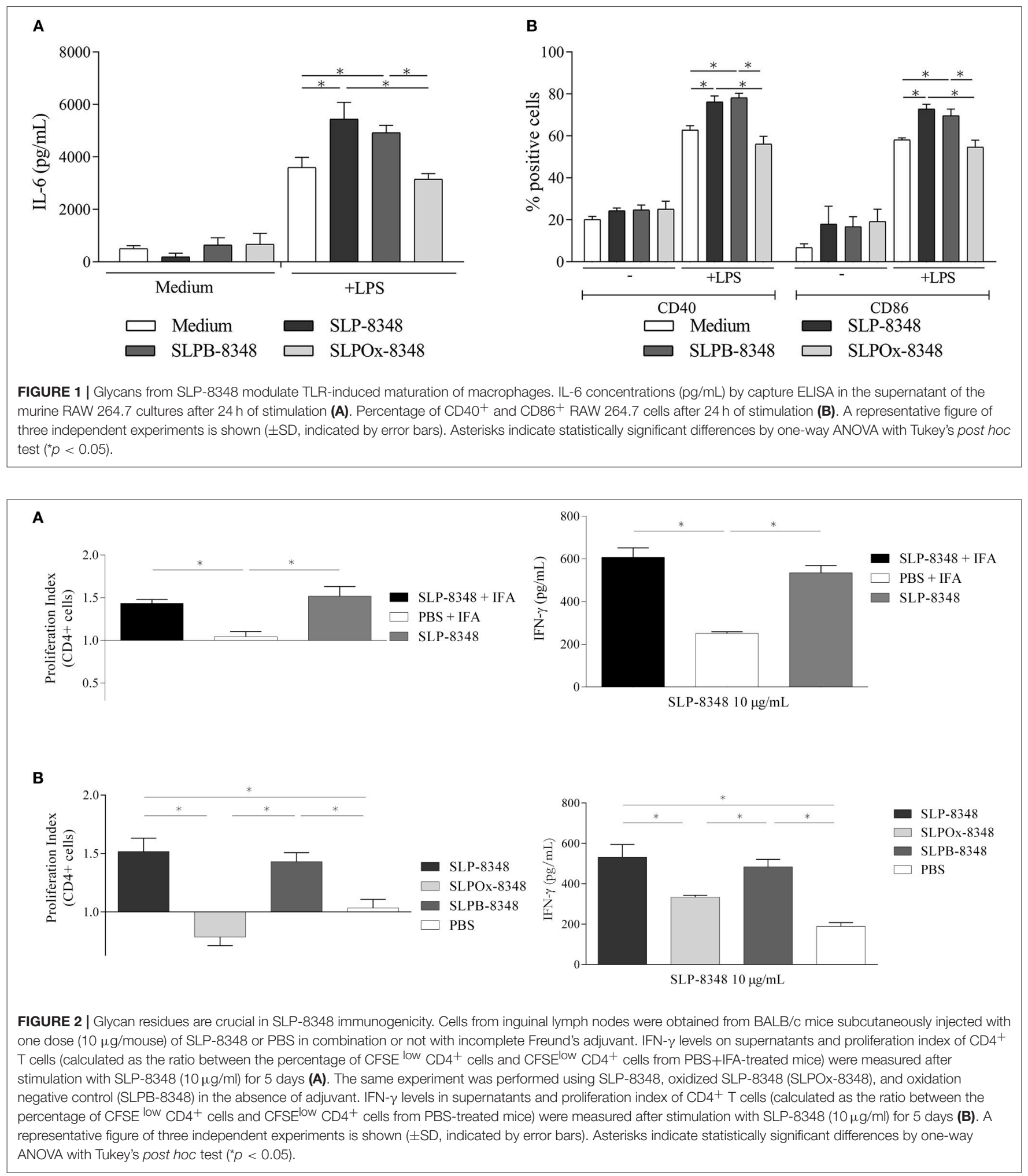

incubation. Additionally, a $\sim 50 \%$ decrease was observed in the binding of SLPOx-8348 to the CLR-hFc fusion proteins compared to SLP-8348 (Supplementary Figure 3). All these findings confirm that the glycans present in SLP-8348 participate in the recognition by these CLRs in a $\mathrm{Ca}^{2+}$-dependent manner (Figure 4A).

After establishing the recognition pattern of CLRs on SLP8348, we investigated whether this SLP-8348/CLRs engagement 

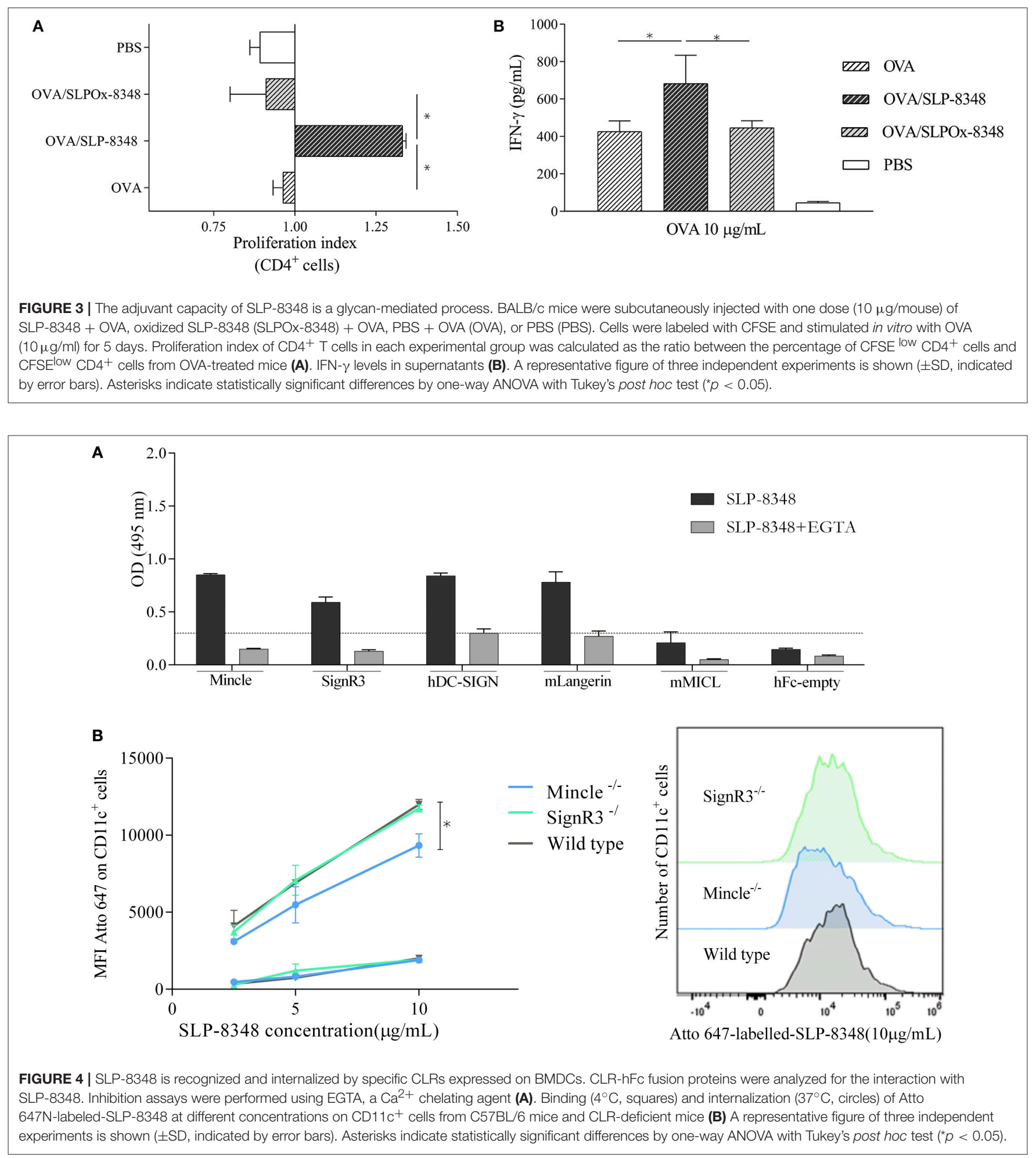

could induce internalization into BMDCs. To this end, Atto647-labeled SLP-8348 was incubated with BMDCs from C57BL/6 wild type mice or CLR-deficient mice both at $4^{\circ} \mathrm{C}$ and $37^{\circ} \mathrm{C}$ and the fluorescence intensity was determined by flow cytometry. As shown in Figure 4B, SLP-8348 both interacted with BMDCs and was internalized by them. We found that SLP-8348 internalization was significantly reduced into BMDCs derived from Mincle ${ }^{-/-}$mice but not from SignR3 ${ }^{-/-}$mice, indicating that the SLP-8348 uptake partially depends on glycan recognition by Mincle. 


\section{SLP-8348 Induce Maturation of BMDCs Through Mincle Recognition}

To address the immunomodulatory properties of SLP-8348, we incubated SLPs with BMDCs from C57BL/6 wild type mice. After $24 \mathrm{~h}$ of incubation, SLP-8348 was able to induce increased levels of IL- 6 and TNF- $\alpha$, as well as the expression of CD40 and CD80 (Figures 5A,B). The same effect was observed using the combination of LPS and SLP-8348, generating higher levels of IL- 6 and TNF- $\alpha$, as well as higher expression of the costimulatory molecules CD40 and CD80 than LPS-stimulated BMDCs (Figures $\mathbf{5 A}, \mathbf{B}$ ).

Given the capacity of Mincle to recognize and internalize SLP-8348, we investigated whether this receptor or its adaptor protein CARD9 mediated the innate response to SLP-8348 in BMDCs. To this end, BMDCs derived from C57BL/6 wild type mice, Mincle-deficient mice, CARD9-deficient mice and SignR3deficient mice were incubated overnight with SLP-8348, LPS or the combination of both. The absence of Mincle or CARD9 signaling on BMDCs resulted in a significantly reduced IL-6 and TNF- $\alpha$ production as well as CD40 and CD80 expression upon SLP-8384 stimulation compared to SignR3 or wild type BMDCs (Figures 5C,D). The same effect was observed using the combination of LPS and SLP-8348, demonstrating that Mincle and its signaling adaptor CARD9 contribute to the stimulation triggered by SLP- 8348 and the modulation elicited by SLP-8348 on LPS-treated BMDCs (Figures 5C,D).

To further investigate whether the enhanced activation elicited by SLP-8348 affected their T-cell stimulatory function, we incubated OVA-treated BMDCs from wild type, $\mathrm{Mincle}^{-/-}$, $\mathrm{CARD}^{-/-}$, and SignR3 ${ }^{-/-}$mice in the presence or absence of SLP-8348 with purified T cells from OT-II mice. Our results showed that SLP-8348 could enhance the OVA-specific T cell proliferation, since the simultaneous stimulation with OVA and SLP-8348 of BMDCs generated a higher IFN- $\gamma$ production as well as higher $\mathrm{CD}^{+} \mathrm{T}$ cell proliferation compared to OVA-treated BMDCs (76.2 vs. $57.0 \%$, respectively) (Figure 6A). Furthermore, the proliferation index of $\mathrm{CD}_{4}^{+} \mathrm{T}$ cell as well as the IFN$\gamma$ levels were significantly reduced when $\mathrm{CD} 4^{+} \mathrm{T}$ cells from OT-II mice were incubated with OVA/SLP-8348-treated BMDCs derived from $\mathrm{Mincle}^{-/-}$mice or CARD9 ${ }^{-/-}$mice, compared to the same treatment using BMDCs derived from wild type or SignR3 ${ }^{-1-}$ mice (Figure 6B). These results clearly indicate that Mincle and CARD9 signaling mediate an enhanced OVA-specific response induced by SLP- 8348 on BMDCs.

\section{DISCUSSION}

Studies of the biological role that carbohydrates play in bacterial glycoproteins have been increasing over time. In fact, the ability to modify proteins by adding carbohydrates was considered exclusive of eukaryotic cells until the appearance of studies on bacterial S-layer glycoproteins (27-29). The attention that these proteins have gained derives from their exceptional physicochemical properties rendering them a unique organizational structure with high application potential in different areas of modern nanobiotechnology. Taking into account that in our previous report we demonstrated that SLP8348 could enhance the proinflammatory response on LPSstimulated macrophages in a $\mathrm{Ca}^{+2}$-dependent manner (21), we first wanted to analyse the role of the carbohydrates in this process. In this respect, the chemical oxidation of terminal glycans has been probed as an adequate strategy trying to understand the role of glycans in the regulation of host immunity (24). When SLP-8348 was treated with metaperiodate and used to stimulate LPS-treated macrophages, the levels of secreted IL-6 as well as the expression of CD40 and CD80 decreased to those corresponding to the stimulation with LPS alone, indicating that the carbohydrates present on SLP-8348 modulate the TLR-induced maturation of macrophages.

In order to characterize more in detail the immunological properties of SLP-8348, we focused on the study of the antigen specific T-cell proliferation after a single subcutaneous inoculation of SLP-8348. Our results demonstrate the capacity of SLP-8348 to induce a high T-cell proliferation and the production of IFN- $\gamma$, either in the presence or absence of adjuvant. The immunogenicity of a Lactobacilli SLP was also demonstrated by Kajikawa and colleagues, who showed that after repeated high dose intragastric immunization with $L$. acidophilus NCFM, specific antibodies against SlpA were generated (30). To gain insight in the SLP-8348 glycan-mediated immune response in vivo, we took advantage of the chemical oxidation approach and performed the same experiments with SLPOx8348. Our results clearly demonstrate that the immunogenicity of SLP-8348 is based on the carbohydrates linked to the glycoprotein, since the re-stimulation of inguinal lymph nodes derived-cells of SLPOx-8348-treated mice with purified SLP8348 induced lower levels of IFN- $\gamma$ and lower proliferation index of $\mathrm{CD} 4^{+} \mathrm{T}$ cells compared to SLP-8348-treated mice. This role of the glycan structures on the immune response elicited by bacterial glycoproteins was also demonstrated by Horn and colleagues, who showed that the immune stimulation elicited by mannosylated Apa glycoproteins of Mycobacterium spp. relies on the presence of the glycan component and also on the extent of glycosylation (31).

Vaccine adjuvants have traditionally been defined as materials that enhance the immune response to vaccine antigens (32). Beyond the mechanism of action, it appears that adjuvants activate innate immune responses to create a local immunocompetent environment at the injection site and draining lymph nodes, that is required to enhance adaptive immunity to the coadministered antigen $(32,33)$. In accordance with our results, SLP-8348 was able to generate a cell-mediated immune response in the draining lymph node after a subcutaneous injection. Therefore, we decided to evaluate the in vivo adjuvant capacity of SLP-8348 using OVA as model antigen. In OVA/SLP-8348immunized mice, the expansion of OVA-specific $\mathrm{T}$ cells and the production of IFN- $\gamma$ were significantly higher than in the group of mice immunized with OVA alone, showing that SLP8348 could enhance the activation and differentiation of $\mathrm{T}$ cells. This adjuvant capacity of SLP-8348 was also dependent on the biological activity of the SLP-8348 glycans, since the co-administration of OVA and metaperiodate treated-SLP-8348 


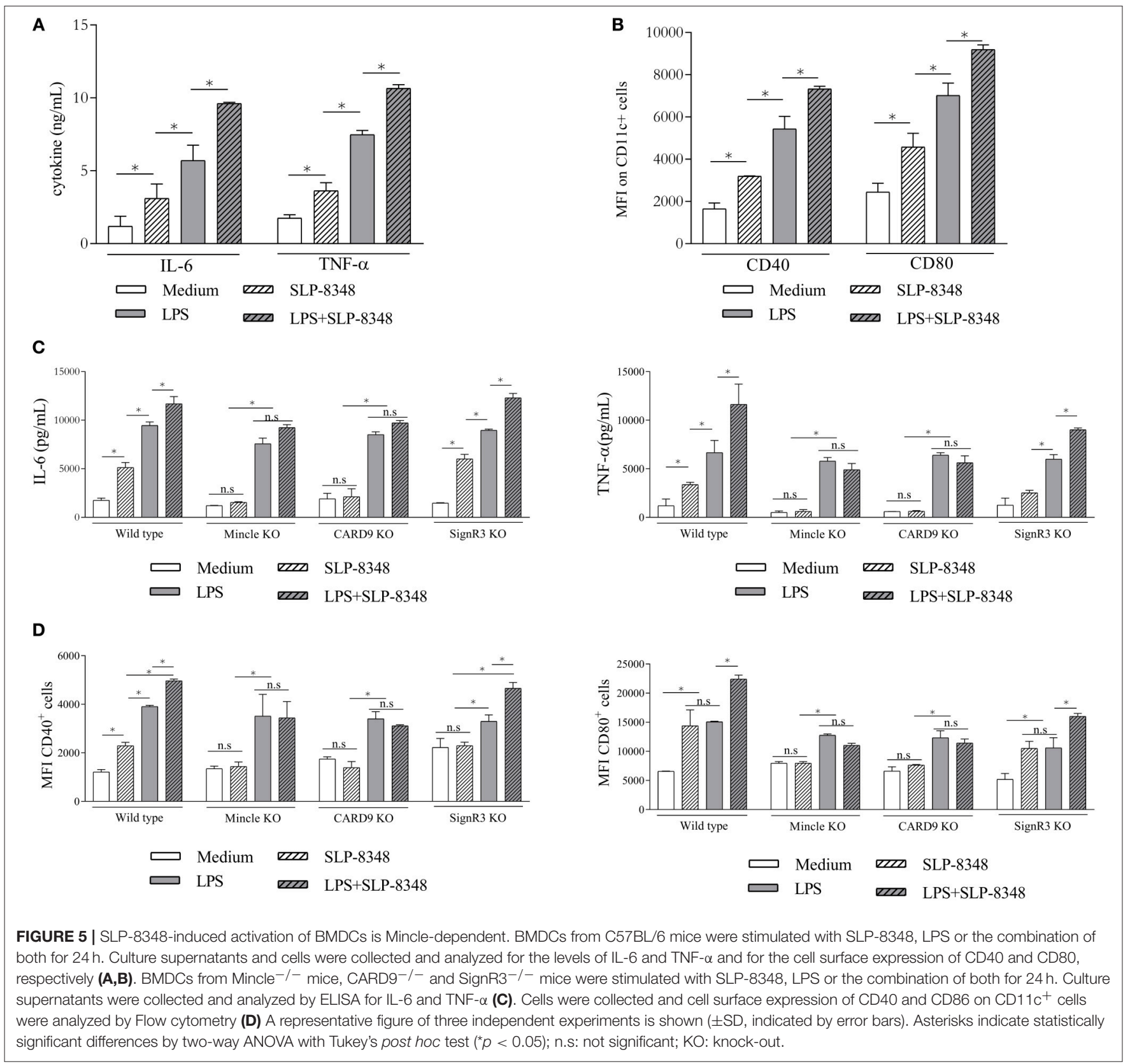

induced a similar adaptive cellular immune response as the administration of OVA alone. Even though previous studies have demonstrated the adjuvant capacity of SLPs from Lactobacillus strains (34), this is the first report showing the real participation of the glycan moieties since the loss of the integrity of terminal glycans completely abrogated the effect. Further studies will be needed in order to test the potential antigenicity of the SLP-8348 in this immunization protocol since it could affect its adjuvanticity.

The evaluation of the adjuvant mechanism of action is a crucial step in the development of effective human vaccines. In this respect, the important role of the glycans in the adjuvant capacity of SLP-8348 led us to study the CLRs that could recognize this glycoprotein. The glycan moieties expressed on SLP-8348 were strongly recognized by the macrophage inducible C-type lectin receptor (Mincle) and to a lesser extent by human DC-SIGN and its murine ortholog SignR3. It was previously reported that binding of SlpA from $L$. acidophilus NCFM to DC-SIGN on human BMDCs (18) or to murine SignR3 (35) is responsible for induction of immunoregulatory signals triggered by the SLP. Also, it was recently reported that Mincle is involved in both the recognition and the immune modulation elicited by the SLP from the oral pathogen Tannerella forsythia (36). Since the ELISAbased method used to detect the binding of SLP-8348 with CLR-Fc fusion proteins can lead to false-positive results, it 


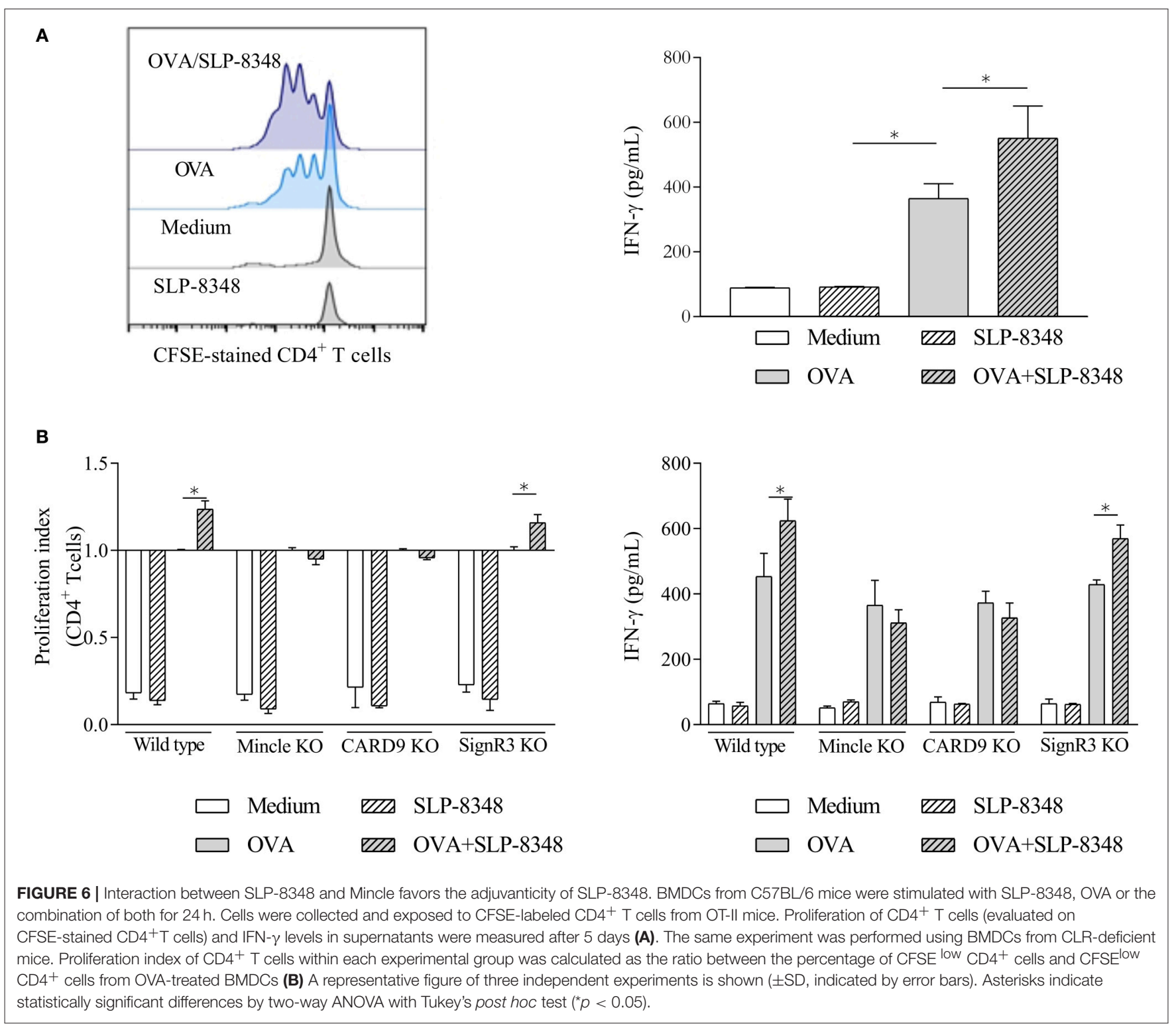

requires confirmation by additional methods (26). Therefore, after establishing the recognition pattern of CLRs on SLP8348 we investigated the biological role of the SLP-8348/CLRs engagement. Our results indicate that BMDCs could internalize SLP-8348 in a Mincle-dependent fashion, since the absence of this receptor on the cell surface of BMDCs reduced the uptake of SLP-8348. In contrast, although SignR3 recognize SLP8348 glycans, this CLR does not mediate the internalization of the glycoprotein.

To elucidate the mechanism that could explain the adjuvant capacity of SLP-8348 we performed in vitro studies employing GM-CSF derived BMDCs which, according to the literature, represent a mixture of DCs, macrophages, and (few) granulocytes (37). SLP-8348 was able to induce a maturation of BMDCs from wild type mice, with production of IL-6, TNF- $\alpha$ and the upregulation of the co-stimulatory molecules CD80 and CD40. When BMDCs were exposed to a simultaneous stimulation with SLP-8348 and LPS, we observed an increased response compared to LPS-stimulated cells. These results partially agree with our previous results, since SLP-8348 did not induce a macrophage activation using RAW 264.7 cells by itself but could enhance the response to stimulation with $E$. coli LPS (21), showing that the same stimulus could trigger different responses depending on the cell type, origin and polarization (38). We demonstrate that SLP-8348 induced maturation of BMDCs in a Mincle-dependent process, since the lack of this receptor generated a significant reduction of the levels of IL-6, TNF- $\alpha$ and co-stimulatory molecules. In addition, our results indicate that CARD9 is also involved in the proinflammatory response elicited by SLP8348 on BMDCs. CARD9 is a caspase recruitment domaincontaining signaling protein that is involved in the signaling pathway of ITAM-coupled receptors in myeloid cells, such as Mincle (39-41). Previous studies have shown that the stimulation of macrophages with the mycobacterial glycolipid trehalose-6,6' dimycolate (TDM), a well-known ligand of Mincle, leads to NF- $\kappa \mathrm{B}$ activation via the $\mathrm{FcR} \gamma / \mathrm{Syk} / \mathrm{CARD} 9$ pathway and results 
in production of proinflammatory cytokines and chemokines such as TNF- $\alpha$, CXCL2, CXCL1, and IL-6 $(41,42)$. Moreover, Schoenen et al have demonstrated that Mincle is necessary for the induction of adaptive immunity using a synthetic analog of TDM as an adjuvant (42). More recently, Shah et al. showed that a synthetic glycolipid (analog to the GL1 from Lactobacillus plantarum) is able to signal through Mincle using a NFATGFP reporter cell line (43). By carrying out OVA-specific Tcell proliferation assays using BMDCs from Mincle-deficient and CARD9-deficient mice, we demonstrate that Mincle and its signaling adaptor CARD9 mediate the adjuvant capacity of SLP8348. CARD9 is involved in the signaling pathway of different CLRs (39). The phenocopy of $\mathrm{Mincle}^{-/-}$and $\mathrm{CARD9}^{-/-}$ BMDCs strongly suggests that Mincle is a CLR that is involved in the immunomodulatory activity of SLP-8348. It has been shown that Mincle can interact with ligands of diverse chemical origin (43-46). In this regard, further studies are needed to determine the glycan structure of L. kefiri CIDCA 8348, in order to characterize the nature of the carbohydrate residues specifically recognized by Mincle.

In conclusion, we have demonstrated that SLP from L. kefiri CIDCA 8348 is able to enhance the OVA-specific immune response by triggering maturation of antigen presenting cells through the recognition of glycan moieties by Mincle. These results constitute a significant contribution to the understanding of the role of glycans in the immunomodulation elicited by bacterial SLPs and generate a promising opportunity in the search for new adjuvants derived from non-pathogenic microorganisms.

\section{DATA AVAILABILITY}

The raw data supporting the conclusions of this manuscript will be made available by the authors, without undue reservation, to any qualified researcher.

\section{ETHICS STATEMENT}

This study was carried out in accordance with the recommendations of the Institutional Animal Care and

\section{REFERENCES}

1. Sleytr UB, Schuster B, Egelseer EM, Pum D. S-layers: principles and applications. FEMS Microbiol Rev. (2014) 38:82364. doi: 10.1111/1574-6976.12063

2. Sára M, Sleytr UB. S-layer proteins. J Bacteriol. (2000)182:85968. doi: 10.1128/JB.182.4.859-868.2000

3. Hynonen U, Palva A. Lactobacillus surface layer proteins: structure, function and applications. Appl Microbiol Biotechnol. (2013) 97:5225243. doi: $10.1007 / \mathrm{s} 00253-013-4962-2$

4. Pum D, Toca-Herrera JL, Sleytr UB. S-layer protein self-assembly. Int J Mol Sci. (2013) 14:2484-501. doi: 10.3390/ijms14022484

5. Takeuchi O, Akira S. Pattern recognition receptors and inflammation. Cell. (2010) 140:805-20 doi: 10.1016/j.cell.2010.01.022

6. Kawasaki T, Kawai T. Toll-like receptor signaling pathways. Front Immunol. (2014) 5:461. doi: 10.3389/fimmu.2014.00461
Use Committee of the Facultad de Ciencias Exactas, Universidad Nacional de La Plata. The protocol was approved by the same committee (Protocol 006-00-18).

\section{AUTHOR CONTRIBUTIONS}

MM performed the experiments, analyzed data, and prepared the manuscript. PC contributed with mice immunizations and flow cytometry experiments. MA participated in SLPs purification and mice immunizations. TF contributed with reagents, designed and analyzed the experiments involving SLPOx-8348, and revised the manuscript. BL and MS conceived the work, analyzed data, and revised the manuscript.

\section{FUNDING}

This study was supported by the Agencia Nacional de Promoción Científica y Tecnológica (ANPCyT) (PICT 2016-0244), Universidad Nacional Arturo Jauretche (UNAJ) (Project 80020170100031 UJ) (Argentina). TF received funding by Agencia Nacional de Innovación e Investigación (ANII, FCE1_2017_1_136094 and MOV_CO_2014_1_103132) and Comisión Sectorial de Investigación Científica (CSIC-I+D-114). $\mathrm{MM}$ was also supported by funding from the German Academic Exchange Service (DAAD) and Asociación de Universidades Grupo Montevideo (AUGM). This publication was supported by Deutsche Forschungsgemeinschaft and University of Veterinary Medicine Hannover, Foundation, within the funding programme Open Access Publishing.

\section{ACKNOWLEDGMENTS}

We thank João T. Monteiro for help with the CLR binding studies and $\mathrm{BMDC} / \mathrm{T}$ cell co-culture assay.

\section{SUPPLEMENTARY MATERIAL}

The Supplementary Material for this article can be found online at: https://www.frontiersin.org/articles/10.3389/fimmu. 2019.01422/full\#supplementary-material
7. Gornati L, Zanoni I, Granucci F. Dendritic cells in the cross hair for the generation of tailored vaccines. Front Immunol. (2018) 9:1484. doi: 10.3389/fimmu.2018.01484

8. Itano AA, McSorley SJ, Reinhardt RL, Ehst BD, Ingulli E, Rudensky AY, et al. Distinct dendritic cell populations sequentially present antigen to CD4 T cells and stimulate different aspects of cell-mediated immunity. Immunity. (2003)19:47-57. doi: 10.1016/S1074-7613(03)00175-4

9. Garrote GL, Delfederico L, Bibiloni R, Abraham AG, Perez PF, Semorile L, et al. Lactobacilli isolated from kefir grains: evidence of the presence of S-layer proteins. J Dairy Res. (2004) 71:222-30. doi: 10.1017/S0022029904000160

10. Mobili P, Serradell MA, Trejo SA, Aviles Puigvert FX, Abraham AG, De Antoni GL. Heterogeneity of S-layer proteins from aggregating and nonaggregaing Lactobacillus kefir strains. Antonie Van Leeuwenhoek. (2009) 95:363-72. doi: 10.1007/s10482-009-9322-y

11. Malamud M, Carasi P, Bronsoms S, Trejo SA, Serradell ML. Lactobacillus kefiri shows inter-strain variations in the amino acid 
sequence of the S-layer proteins. Antonie Van Leeuwenhoek. (2017) 110:515-30. doi: 10.1007/s10482-016-0820-4

12. Carasi P, Ambrosis NM, De Antoni GL, Bressollier P, Urdaci MC, Serradell MA. Adhesion properties of potentially probiotic Lactobacillus kefiri to gastrointestinal mucus. J Dairy Res. (2014) 81:16-23. doi: 10.1017/S0022029913000526

13. Zubiría MG, Gambaro SE, Rey MA, Carasi P, Serradell MA, Giovambattista A. Deleterious effects of high-fructose intake: the preventive effect of Lactobacillus kefiri administration. Nutrients. (2017) 9:470. doi: 10.3390/nu9050470

14. Carasi P, Trejo FM, Perez PF, de Antoni GL, Serradell M. Surface proteins from Lactobacillus kefiri. antagonize in vitro cytotoxic effect of Clostridium difficile toxins. Anaerobe. (2012) 18:135-9. doi: 10.1016/j.anaerobe.2011.11.002

15. Golowczyc MA, Mobili P, Garrote GL, Abraham AG, De Antoni GL. Protective action of Lactobacillus kefir carrying S-layer protein against Salmonella enterica serovar Enteritidis. Int J Food Microbiol. (2007) 118:26473. doi: 10.1016/j.ijfoodmicro.2007.07.042

16. Carasi P, Díaz M, Racedo de Antoni GL, Urdaci MC, Serradell MA. Safety characterization and antimicrobial properties of kefir-isolated Lactobacillus kefiri. Biomed Res Int. (2014) 2014:208974. doi: 10.1155/2014/208974

17. Schäffer C. Surface-layer glycoproteins: an example for the diversity of bacterial glycosylation with promising impacts on nanobiotechnology. Glycobiology. (2004)14:31R-42R. doi: 10.1093/glycob/cwh064

18. Konstantinov SR, Smidt H, de Vos WM, Bruijns SC, Singh SK, Valence F, et al. S layer protein A of Lactobacillus acidophilus NCFM regulates immature dendritic cell and T cell functions. Proc Natl Acad Sci USA. (2008) 105:194749. doi: 10.1073/pnas.0810305105

19. Liu Z, Ma Y, Shen T, Chen H, Zhou Y, Zhang P, et al. Identification of DC-SIGN as the receptor during the interaction of Lactobacillus plantarum CGMCC 1258 and dendritic cells. World J Microbiol Biotechnol. (2011) 27:603-11. doi: 10.1007/s11274-010-0495-3

20. Prado Acosta M, Ruzal SM, Cordo SM. S-layer proteins from Lactobacillus sp. inhibit bacterial infection by blockage of DC-SIGN cell receptor. Int J Biol Macromol. (2016) 92:998-1005. doi: 10.1016/j.ijbiomac.2016.07.096

21. Malamud M, Carasi P, Freire T, Serradell MLA. S-layer glycoprotein from Lactobacillus kefiri CIDCA 8348 enhances macrophages response to LPS in $\mathrm{a} \mathrm{Ca}^{+2}$-dependent manner. Biochem Biophys Res Commun. (2018) 495:122732. doi: 10.1016/j.bbrc.2017.11.127

22. Garrote GL, Abraham AG, De Antoni GL. Chemical and microbiological characterisation of kefir grains. J Dairy Res. (2001) 68:639-52. doi: 10.1017/S0022029901005210

23. Laemmli UK. Cleavage of structural proteins during the assembly of the head of bacteriophage T4. Nature. (1970) 227:680-5. doi: 10.1038/227680a0

24. Rodríguez E, Noya V, Cervi L, Chiribao ML, Brossard N, Chiale C, et al. Glycans from fasciola hepatica modulate the host immune response and TLR-induced maturation of dendritic cells. PLoS Negl Trop Dis. (2015) 9:e0004234. doi: 10.1371/journal.pntd.0004234

25. Bradford M. A rapid and sensitive method for the quantitation of microgram quantities of protein utilizing the principle of protein-dye binding. Anal Biochem. (1976) 72:248-54. doi: 10.1016/0003-2697(76)90527-3

26. Mayer S, Moeller R, Monteiro JT, Ellrott K, Josenhans C, Lepenies B. Ctype lectin receptor (CLR)-Fc fusion proteins as tools to screen for novel CLR/bacteria interactions: an exemplary study on preselected Campylobacter jejuni isolates. Front Immunol. (2018) 9:213. doi: 10.3389/fimmu.2018.00213

27. Mescher MF, Strominger JL, Watson SW. Protein and carbohydrate composition of the cell envelope of Halobacterium salinarium. J Bacteriol. (1974) 120:945-54.

28. Sleytr UB, Thorne KJ. Chemical characterization of the regularly arranged surface layers of Clostridium thermosaccharolyticum and Clostridium thermohydrosulfuricum. J Bacteriol. (1976) 126:377-83.

29. Comstock and Kasper. Bacterial glycans: key mediators of diverse host immune responses. Cell. (2006) 126:847-50. doi: 10.1016/j.cell.2006.08.021

30. Kajikawa A, Zhang L, LaVoy A, Bumgardner S, Klaenhammer TR, Dean GA. Mucosal immunogenicity of genetically modified Lactobacillus acidophilus expressing an HIV-1 epitope within the surface layer protein. PLoS ONE. (2015) 10:e0141713. doi: 10.1371/journal.pone.0141713

31. Horn C, Namane A, Pescher P, Riviere M, Romain F, Puzo G, et al. Decreased capacity of recombinant $45 / 47-\mathrm{kDa}$ molecules (Apa) of
Mycobacterium tuberculosis to stimulate $\mathrm{T}$ lymphocyte responses related to changes in their mannosylation pattern J Biol Chem. (1999) 274:32023-30. doi: 10.1074/jbc.274.45.32023

32. O'Hagan T, Friedland L, Hanon E, Didierlaurent A. Towards an evidencebased approach for the development of adjuvanted vaccines. Curr Opin Immunol. (2017) 47:93-102 doi: 10.1016/j.coi.2017.07.010

33. Awate S, Babiuk L, Mutwiri G. Mechanisms of action of adjuvants. Front Immunol. (2013) 4:114. doi: 10.3389/fimmu.2013.00114

34. Anzengruber J, Bublin M, Bönisch E, Janesch B, Tscheppe A, Braun ML, et al. Lactobacillus buchneri S-layer as carrier for an Ara h 2-derived peptide for peanut allergen-specific immunotherapy. Mol Immunol. (2017) 85:818. doi: 10.1016/j.molimm.2017.02.005

35. Lightfoot YL, Selle K, Yang T, Goh YJ, Sahay B, Zadeh M, et al. SIGNR3dependent immune regulation by Lactobacillus acidophilus surface layer protein A in colitis. EMBO J. (2015) 34:881-95. doi: 10.15252/embj.201490296

36. Chinthamani S, Settem RP, Honma K, Kay JG, Sharma A. Macrophage inducible C-type lectin (Mincle) recognizes glycosylated surface (S)- layer of the periodontal pathogen Tannerella forsythia. PLoS ONE. (2017) 12:e0173394. doi: 10.1371/journal.pone.0173394

37. Helft J, Böttcher J, Chakravarty P, Zelenay S, Huotari J, Schraml BU, et al. GM-CSF mouse bone marrow cultures comprise a heterogeneous population of CD11c+MHCII+ macrophages and dendritic cells. Immunity. (2015) 42:1197-211. doi: 10.1016/j.immuni.2015.05.018

38. Habil N, Al-Murrani W, Beal J, Foey AD. Probiotic bacterial strains differentially modulate macrophage cytokine production in a straindependent and cell subset-specific manner. Benef Microbes. (2011) 2:28393. doi: 10.3920/BM2011.0027

39. Ruland. CARD9 signaling in the innate immune response. Ann N Y Acad Sci. (2008) 1143:35-44. doi: 10.1196/annals.1443.024

40. Sancho D, Reis e Sousa C. Signaling by myeloid C-type lectin receptors in immunity and homeostasis. Annu Rev Immunol. (2012) 30:491529. doi: 10.1146/annurev-immunol-031210-101352

41. Ishikawa E, Ishikawa T, Morita Y, Toyonaga K, Yamada H, Takeuchi O, et al Direct recognition of the mycobacterial glycolipid, trehalose dimycolate, by Ctype lectin Mincle. J Exp Med. (2009) 206:2879-88. doi: 10.1084/jem.20091750

42. Schoenen H, Bodendorfer B, Hitchens K, Manzanero S, Werninghaus $\mathrm{K}$, Nimmerjahn $\mathrm{F}$, et al. Cutting edge: mincle is essential for recognition and adjuvanticity of the mycobacterial cord factor and its synthetic analog trehalose-dibehenate. J Immunol. (2010) 184:2756-60. doi: 10.4049/jimmunol.0904013

43. Shah S, Nagata M, Yamasaki S, Williams SJ. Total synthesis of a cyclopropane-fatty acid $\alpha$-glucosyl diglyceride from Lactobacillus plantarum and identification of its ability to signal through Mincle. Chem Commun. (2016) 52:10902-5. doi: 10.1039/C6CC05631H

44. Yamasaki S, Ishikawa E, Sakuma M, Hara H, Ogata K, Saito, T. Mincle is an ITAM-coupled activating receptor that senses damaged cells. Nat Immunol. (2008) 9:1179-88. doi: 10.1038/ni.1651

45. Wells CA, Salvage-Jones JA, Li X, Hitchens K, Butcher S, Murray RZ, et al. The macrophage-inducible C-type lectin, mincle, is an essential component of the innate immune response to Candida albicans. J Immunol. (2008) 180:7404-13. doi: 10.4049/jimmunol.180.11.7404

46. Yamasaki SM, Matsumoto O, Takeuchi T, Matsuzawa E, Ishikawa $M$, Sakuma $H$, et al. C-type lectin Mincle is an activating receptor for pathogenic fungus, Malassezia. Proc Natl Acad Sci USA. (2009) 106:1897-902. doi: 10.1073/pnas.0805 177106

Conflict of Interest Statement: The authors declare that the research was conducted in the absence of any commercial or financial relationships that could be construed as a potential conflict of interest.

Copyright () 2019 Malamud, Carasi, Assandri, Freire, Lepenies and Serradell. This is an open-access article distributed under the terms of the Creative Commons Attribution License (CC BY). The use, distribution or reproduction in other forums is permitted, provided the original author(s) and the copyright owner(s) are credited and that the original publication in this journal is cited, in accordance with accepted academic practice. No use, distribution or reproduction is permitted which does not comply with these terms. 